\title{
The neurological dynamics of the imagination
}

\author{
John Kaag
}

(C) Springer Science + Business Media B.V. 2008

\begin{abstract}
This article examines the imagination by way of various studies in cognitive science. It opens by examining the neural correlates of bodily metaphors. It assumes a basic knowledge of metaphor studies, or the primary finding that has emerged from this field: that large swathes of human conceptualization are structured by bodily relations. I examine the neural correlates of metaphor, concentrating on the relation between the sensory motor cortices and linguistic conceptualization. This discussion, however, leaves many questions unanswered. If it is the case that the sensory motor cortices are appropriated in language acquisition, how does this process occur at the neural level? What neural preconditions exist such that this appropriation is possible? It is with these questions in mind that I will turn my attention to studies of neural plasticity, degeneracy and the mirror neuron activation. Whereas some scholarship in philosophy and cognitive neuroscience has aimed to identify the neurological correlates of consciousness, examining plasticity, degeneracy and activation shifts the discussion away from a study of correlates toward an exploration of the neurological dynamics of thought. This shift seems appropriate if we are to examine the processes of the "imagination."
\end{abstract}

Keywords Imagination $\cdot$ Metaphor $\cdot$ Mirror neurons $\cdot$ Plasticity $\cdot$ Degeneracy

\section{Introduction}

The imagination is difficult to define. More often than not, it is not defined at allonly invoked as a placeholder by philosophers when they are unable to define particular cognitive processes. When Kant explored the relation between the body and the mind in the Critique of Pure Reason, the imagination was called in to mediate between the empirical content of the sensuous world and the "pure

J. Kaag $(\bowtie)$

American Academy of Arts and Sciences, Harvard University, 136 Irving Street,

Cambridge, MA 02138, USA

e-mail: jkaag@fas.harvard.edu 
concepts" of the understanding. When he explored the nature of human creativity in the Critique of Aesthetic Judgment, the imagination was once again enlisted to "explain" the way that human organisms might negotiate novel circumstances and generate original aesthetic ideas. The imagination, as a concept, has repeatedly been used to set the boundary between explanation and speculation, and it continues to be invoked when nothing more can be said in regard to human creativity and the embodiment of thought and language. One of the tasks shared by phenomenology and cognitive neuroscience is to explore the borderland that the "imagination" has demarcated. Proust does not suggest that "the imagination grants the possibility of Remembrance of Things Past," but rather draws a reader into the place of the "imagination" and invites a closer look at the phenomenon and event that has often been glossed by this term. Similarly, contemporary cognitive neuroscience sheds new light on questions that the "imagination" outlined: the question of mind and body, the question of novel creativity, the phenomenon of adaptation and plasticity.

This article examines the imagination by way of various studies in cognitive science. It opens by examining the neural correlates of bodily metaphors. It assumes a basic knowledge of metaphor studies, or the primary finding that has emerged from this field: that large swathes of human conceptualization are structured by bodily relations. I examine the neural correlates of metaphor, concentrating on the relation between the sensory motor cortices and linguistic conceptualization. But why begin with metaphor? In Kant's critical project, the imagination was regarded as the bridge between the sensorial and the conceptual realms (this is the function of Kant's "schematism"); this mediating process was regarded as the imagination's "reproductive" and "productive" function. The current study of conceptual metaphor investigates these functions of the imagination by exposing the way in which "higher" forms of human abstraction are rooted in body experience. This discussion, however, leaves many questions unanswered. If it is the case that the sensory motor cortices are appropriated in language acquisition, how does this process occur at the neural level? What neural preconditions exist such that this appropriation is possible? It is with these questions in mind that I will turn my attention to studies of neural plasticity, degeneracy and the mirror neuron activation. Whereas some scholarship in philosophy and cognitive neuroscience has aimed to identify the neurological correlates of consciousness, examining plasticity, degeneracy and activation shifts the discussion away from a study of correlates toward an exploration of the neurological dynamics of thought. This shift seems appropriate and necessary if we are to examine the processes of the "imagination."

The imagination, in its reproductive and productive roles, has been described in the history of philosophy as the connecting link between sensation and conception, effectively but ambiguously spanning the mind-body divide. It has also, and perhaps more importantly, been regarded as the dynamic process by which organisms (and more particularly humans) negotiate their ever-changing circumstances by way of the creative powers of mind. The investigation of neural plasticity, degeneracy and the mirror neuron system is the attempt to partially sketch the developmental and organic basis of these creative powers of mind. The creativity that we identify at the level of the human thought and action-which since the Enlightenment has been regarded as adaptive, non-deterministic, and free-proves to be isomorphic with the complex dynamics of the human morphology. 
In this article, I do not regard the imagination as a purely "artistic" faculty enjoyed by a select handful of inspired savants. To do so would be to follow the lead of many thinkers in the history of philosophy, but to follow these thinkers into an unproductive cul de sac. Instead I take the imagination to refer to the creative and embodied processes of mind that are common to human beings on the whole and that are necessary to "get on with our business" in our social and natural surroundings.

At first glance, this project might appear as the search for the bodily origins of human creativity. First glances can be both accurate and deceiving. It is accurate to say that the current study aims to provide a description of creativity that attends to the embodied nature of human organisms. It is also accurate to say that it stands against accounts of aesthetic creation and epistemology that rest upon a disembodied conception of mind. Attending to embodiment, however, is not to reduce the imagination to the descriptions of a deterministic physicalism or eliminative materialism. ${ }^{1}$ No one biological "origin" can exhaustively account for the variety of forms of conceptual and artistic novelty. This fact, does not force us to abandon cognitive science, but rather ought to encourage us to make our science as nimble as our phenomenology. Indeed, this study calls for a scientific paradigm, defined by the recognition of complexity and indeterminacy, which surpasses a simple materialism without succumbing to the élan vital of naïve idealism.

\section{Coming to our senses: Affect and the body of thought}

In recent years, convergent evidence from the cognitive neurosciences has pointed to the neural and bodily basis of metaphor and suggested that image schemata ought to be considered "dynamic activation patterns that are shared across the neural maps of the sensory motor cortex" (Rohrer 2006, p. 72). More plainly, the evidence has begun to show that the brain is fundamentally multi-modal and cross-modal. This evidence will be addressed first by way of broad strokes, and then by more detailed accounts of neural development, architecture, and function that begin to describe the embodied basis of imaginative thought.

Lakoff and Johnson repeatedly underscores the multimodality of actions, highlighting the way in which motor, perceptual, and somato-sensory components are coordinated. For example, these components allow an individual to respectively do an action, to perceive the action being done, and to "get the sense" of doing the action. This coordination is reflected in neural activation patterns, the study of which gave rise to the hypothesis that multimodal coordination might ground abstract thought. After exploring this hypothesis, researchers found that there is a simultaneous coordination of different neural domains that underpin the mapping between the metaphoric domains that Lakoff and Johnson began to identify in the 1980s. Specifically, recent work has indicated that there is a continual coordination

\footnotetext{
${ }^{1}$ Daniel Dennett has been accused of this type of eliminative materialism which states that a discussion of mental dispositions such as qualia or the imagination ought to be cut short in favor of a detailed materialist account of mind. See Dennett (1988). Varela carefully states the dangers of materialism and physicalism. He also outlines the dangers of dismissing cognitive science whole cloth. See Varela (1992, p. 13).
} 
between the sensory-motor domains and the neural domains that have long been regarded as the seat of abstract conceptualization. This neural multimodality has come to the fore in the study of cognitive linguistics.

Debunking the long-held position that Broca's and Wernicke's areas were the exclusive neural loci of semantic understanding and language production, studies have shown that the sensorimotor cortices are crucial to semantic understanding of bodily action terms and sentences (Rose 1987; Glenberg 2002; Hauk 2004; Kohler et al. 2002). In his recent meta-analysis of metaphoric cognition, Timothy Rohrer employs contemporary functional magnetic resonance imaging (fMRI) and eventrelated potentials (ERP) experiments to highlight the way literal and metaphoric stimuli activate areas in the sensorimotor cortex that are consistent with the image schemata hypothesis. In these studies Rohrer first attempts to show that brain areas that researchers once assumed were only activated by spatial and bodily orientations are also activated by linguistic cues that describe these particular orientations. The neural activation detected when one picks up a box is largely isomorphic with the activation stimulated by the command to "pick up that box." Secondly, and more significantly for our study of metaphor and image schema, Rohrer discovers that describing particular physical orientations serves as a literal cue that generates activation patterns which are largely isomorphic with the patterns detected when a subject is exposed to polysemous cues, cues or signs with multiple meanings. For example, the literal expression "pick that box up" activates the same neural pattern as the metaphoric or polysemic expressions such as "turn up the volume" or "he turned it up in that basketball game." This result obtained when other schemas, such as the "out" schema discussed earlier, were tested in a similar way. In short, polysemous cues trigger spatial and corporeal relations, indicating that the "mind" that handles abstract conceptualization can, in an important sense, be found in the bodily relations of a human organism. This is not to merely make the claim that one needs a body to think, but rather the stronger claim that our bodies, and their relationship with their environmental situations, continually structure human thinking.

Rohrer notes that image schemas and corresponding neural maps develop over time, evolving to accommodate novel situations and, in this development, actually exhibit a type of emergent creativity of their own. The work of Rohrer indicates that abstract conceptualization appropriated or co-opted the structured neural relations of the sensory motor cortex in the development of the brain architecture that could support abstract cognition.

Gerald Edelman's concept of secondary neural repertoires echoes Rohrer's account, and is a likely neural process to explain how integrative areas of the sensorimotor cortex acquire both sensorimotor and image schematic functions (Edelman 1987b). ${ }^{2}$ A brief description of Edelman's account will outline the degree to which this cross-modal structuring - in its emergent character-obtains in the physical activities of a living organism. More simply, the architecture and dynamics

\footnotetext{
${ }^{2}$ Edelman goes on to describe the particular mechanisms that grant the possibility of the development of secondary functional repertoires in his concept of "reentry" which stands apart from neural "feedback." This distinction and the imaginative character of reentry will be addressed in the discussion of the organic/ molecular basis of the imagination. See Edelman and Tononi (2000b, p. 64).
} 
of the human nervous system is continuous with, and continually structures, the life of the mind and language. In his description of secondary neural repertoires, Edelman helps us explain the possibility of the metaphoric process of the imagination, the mapping of particular image schemas, in highly specific physical processes that demonstrate the similar modes of organization and emergence.

Edelman argues that neuroembryonic development (the development of neural maps) can best be understood as a process he calls Neural Darwinism. First, through the process of cell division, growth and selection, neural sites are established. These sites should be regarded as local neural regions that possess particular patterns of dendritic and axonal arborization determined by morphoregulatory molecules that affect the neural architecture of these particular regions. This developmental selection produces what Edelman calls "primary repertoires" consisting of large numbers of variant neural circuits within particular anatomical regions.

As organic agents, neurons in the embryo seem to flourish, find nourishment, and particular forms of reinforcement-first in developmental selection, then in experiential selection. Neuronal synapses are the units of selection in this model. They undergo, and participate in, a process of neural amplification; in so doing, neural activation patterns make the rather curious journey from chaos to order. As Edelman highlights, this is a form of experiential selection that depends on environmental conditions and the behavior of the organism. Under certain conditions and in light of certain behaviors some neural networks will be activated more than others. The differential activation of neuronal selection serves the same function that differential reproduction serves in natural selection, creating the conditions by which certain networks actively thrive while others become "extinct" or are "pruned." Over time, the neurons that activate in tandem become physically correlated, developing Hebbian associations that engrain and reinforce particular patterns of neural activity. In an overused expression, "neurons that fire together wire together," creating functional clusters that mediate and coordinate the activity of an array of neurons. Through this process, genuine Peircean "thirds" emerge, that is, functional entities irreducible to the two parts between which they meditate. Hebbian association can be described by the function given below (Fig. 1), a function that Peirce roughly approximates in his discussions of human physiology. Here, I provide a more detailed explanation of Hebb's rule:

In developing this function, Hebb sets the groundwork for connectionist theorists in the 1960s who began to create models that envision brain functions to be emergent with global properties resulting from the interaction of connected neural networks. Hebb's rule is also significant in the sense that it identifies growth and metabolic change as the defining features of our nervous system, a feature that coincides with the spontaneity and creativity that has been associated with the imagination. Several points, however, need to be highlighted in order to recognize and qualify the import of Hebb's insight.

First, the metabolic growth $\left(\Delta T_{\mathrm{AB}}\right)$ is calculated as a function of the instantaneous interaction of two individual cells. It is the case that growth depends on the actions and reactions of both cells and emerges as a process that is irreducible to the excitation of either A or B in isolation. What some interpretations of Hebb's rule neglect, however, in their attempt to remain parsimonious, is the fact that these two cells are already interacting in wider neuronal complexes which set the limits and 


\section{Hebb's Rule}

In 1949, Donald Hebb wrote The Organization of Behavior in which he postulates:

"When an axon of cell A is near enough to excite a cell B and repeatedly or persistently takes part in firing it, some growth process or metabolic change takes place in one or both cells such that A's efficiency, as one of the cells firing B, is increased."

This hypothesis, born out in modern cognitive science, is consistent with Peirce's understanding of habit formation, but also point to the way in which novel input stimuli can revise these habits of association. More recently, Byrne translated this postulate into a variety of quantitative expressions in which a neuron $A$ with average firing weight $V_{A}$, projects to neuron $B$ with average firing weight $\mathrm{V}_{\mathrm{B}}$. The synaptic connection from $\mathrm{A}$ to $\mathrm{B}$ has strength $\mathrm{T}_{\mathrm{AB}}$, which determines the degree of activity in $\mathrm{A}$ is capable of exciting in $\mathrm{B}$; the strength of $\mathrm{T}_{\mathrm{AB}}$ should be modified in some way that is dependent on both activity of $\mathrm{A}$ and B. The general expression of this plasticity rule is formulated in the function: ${ }^{7}$

$$
\Delta \mathrm{T}_{\mathrm{AB}}=\mathrm{F}\left(\mathrm{V}_{\mathrm{A}}, \mathrm{V}_{\mathrm{B}}\right)
$$

Fig. 1 Hebbs rule

provide the enabling conditions of the cells' excitation. This limit and enablement arises in the ongoing and non-repeatable transformation of brain-states and metabolic processes. Edelman points to these processes in his description of the creation of secondary repertoires. In emphasizing this point, we can see that Hebb's rule cannot be easily applied to the activation dynamics of neural populations, since these complex dynamics cannot be modeled by a mechanical rule or linear function. I will return to this point in my discussion of neural reentry.

A second point needs to be emphasized in regard to Hebb's rule: The metabolic growth rate is calculated as change at a particular instant in time. This calculation provides only a snapshot or glimpse of neural dynamics without demonstrating the course of events and relations that might have led to this rate of change. That is also to say that the Hebb's rule provides an idealized model of an extremely small time interval of continuous and diverse evolutionary, developmental, and experiential processes. This is not to dismiss Hebb's work, but to encourage us to extend and modify the rule in order to better demonstrate the continuity of neural activation and development.

This qualification of Hebb's rule suggests that neural dynamics should not be regarded as mechanical linear functions. With this being said, the rule also does not support the idea that these dynamics are the products of random chance or pure contingency. To return to a point made earlier, this form of creative irregularity is irreversible and directed. As psychiatrist Jeffrey Shwartz notes in his experiments on language acquisition, "Once the Hebbian process has claimed circuits, they are hardwired for that sound; so far, neuroscientist have not observed any situations in which the Hebbian process is reversed so that someone born into a sea of, say, Finnish losses the ability to hear Finnish unique sounds." (Schwartz and Begley 2002) The metabolic change that occurs is an irrevocable fact that continues to affect-albeit in 
a dissipating or decaying degree - the future transformations in the neural activation of the system. It is in this sense that neural dynamics are motivated by, and arise in, the creative spontaneity that has been outlined in our discussion of the imagination, and in Peirce's description of abduction and tychism.

It is worth noting that Charles Sanders Peirce makes an identical point in "Thinking and Cerebration" in 1879: "Neural activation causes fatigue, but long continued stimulation causes another phenomenon, namely, the spread from cell to cell of the nervous activity...along whatever path a nervous discharge takes place, along that path a new discharge is likely to take place." ${ }^{3}$ This spread of activation causes physical and dynamic adjustments in our neuronal complexes over time that are genuinely novel and irreversible. William James, following in Peirce's footsteps, anticipates Hebb's rule when he writes on the process of mental association in his Psychology in 1890: "the amount of activity at any one point in the brain cortex is the sum of the tendencies of all other points to discharge into it... When two brain processes have been active together in immediate succession, one of them on recurring, tends to propagate the other."(James 1950) In his recent studies, Rohrer elaborates on this point, explaining that neurons aggregate over time in neuronal groups in a process that can be described by rules of plasticity, but, of course, cannot be predicted or anticipated by these rules. These aggregates, in turn, "act like organisms that seek out stimulation as nourishment, and the neuronal groups compete with each other as they migrate along the neural tube toward the emerging sense organs." ${ }^{4}$ This aggregation is describable by way of Hebb's rule and lends credence to the stance of Peirce and James.

The migration and population-growth dynamics of the neuronal groups create yet another emergent property: Neurons array themselves into physical patterns that "map" the various sensory modalities. This mapping refers to the fact, culled from an array of experiments since the 1960s, that particular posterior regions of the brain space are associated with particular sensory functions. More carefully stated, Edelman explains that the use of physical space in the posterior regions of the brain to represent environmental stimuli provides the incipient topographical neural maps of the sensory modalities. To reference topology is appropriate in this case since Gallistel and others have observed the vector spaces of topology have a literal interpretation in the nervous system (Gallistel 1990). An explanation of the following schematic of the development of primary and secondary neural repertoires may help to elucidate this point (Fig. 2).

In phase one of neuronal group selection, developmental selection occurs as a result of growth factor signaling and selective pressures that "yield anatomical networks in each individual." (Edelman 1989) This involves the development of neural sites described earlier and is modeled in part one of the diagram above and provides a primary neural repertoire. Next, "selective strengthening or weakening of particular populations of synapses occurs as a result of behavior." The weighting of particular synaptic activations is represented by the bold lines of connection seen in

\footnotetext{
${ }^{3}$ CP 4.39.

${ }^{4}$ Ibid., 19.

5 Ibid.
} 


\section{Reentry and Neural Darwinism}

1) Developmental Selection

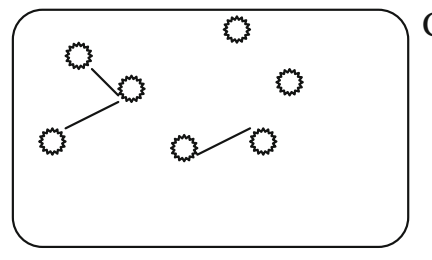

Cell Division

Cell Death

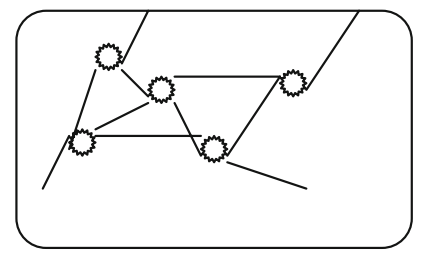

2) Experiential Selection - Hebbian Association

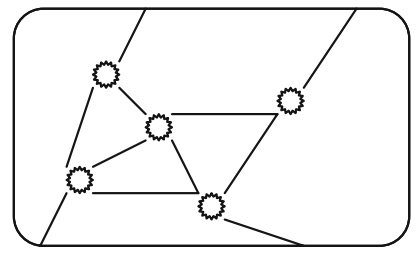

Changes in

Strength of

Synapses

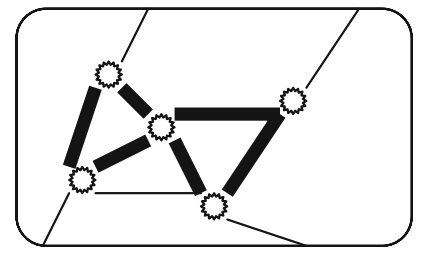

3) Reentrant Mapping

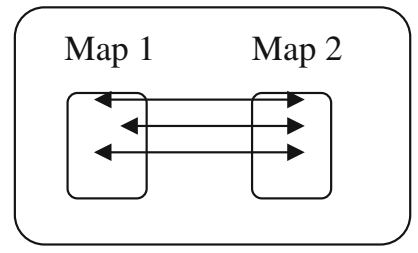

Time 1
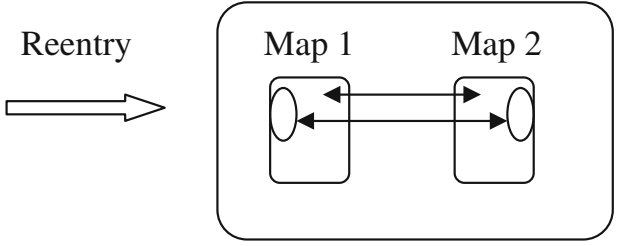

Time 2

Fig. 2 Reentry and neural Darwinism

part two of the above diagram. The weighting of synaptic activation creates a relatively stable organic framework that underpins our relatively stable mental lives. It is worth noting that the Darwinian concepts of propagation, selection, and adaptation - concepts that underpin evolutionary theory-have been imported into Edelman's account of physiological development. It is for this reason that Edelman's theory is often referred to as somatic evolution.

Finally, reentry, a process which we will examined in some detail in the coming sections, coordinates neural maps through "the parallel selection and correlation of neuronal groups in different areas" of the brain that makes possible the emergence of complex sensory and conceptual meanings. ${ }^{6}$ After multiple exposures to a stimulus, activation patterns are established in a variety of mapped areas. "Operations in these different maps that are related to the same perceptual stimulus are linked together by

\footnotetext{
${ }^{6}$ Ibid., 48.

글 Springer
} 
reentry." ${ }^{7}$ An example is helpful in elucidating this last stage of neuronal organization. As Donald Tucker notes, "The primary auditory cortex is mapped by frequency (pitch of the sounds, high or low), not by higher perceptual objects such as words. Therefore, processing at deeper or "higher" levels is required to form the perception of a word that gets meaning from the sounds." The comprehension of a word depends upon heteromodal coordination involving numerous functional maps. This heteromodal coordination and synchronization is what Edelman refers to as reentry.

Cross-modal coordination grants the possibility of the categorization and abstract conceptualization that Roher identified in his fMRI and ERP experiments described earlier. In terms of the investigation of image schematic and metaphoric forms of cognition, it is worth mentioning that auditory areas develop maps indicating increasing pitch and volume in this way. Later, as Rohrer notes, "tactile areas develop somatic maps for pain and touch along limbs; and still later, somatomotor maps develop for muscles distributed across the limbs." 8 The schematic of neural adaptation and selection is deceptive in the sense that the level of analysis shifts between inter-neural dynamics of phase one and phase two and the inter-map dynamics of reentry depicted in phase three of the schematic. This shift is confusing, but necessary in order to present the emergent phenomenon of reentry between developing neural regions.

Rohrer hypothesizes that the synchronization of neural maps is necessary in the establishment of cross-modal image schematic patterns. This is most notable between auditory and tactile neural nodes. It is worth pointing out that the development and coordination of neural maps does not stop in any determinate manner; indeed, this neural development, with its simultaneous propagation of connectivity and diversity, proceeds in a way that cannot be predicted nor exhaustively described, exhibiting a type of plasticity that has been described as uniquely imaginative. ${ }^{9}$

This fact is born out in several studies. For example, in their experiments with primates, Allard et al. has demonstrated that organisms are free to dynamically reorganize the somatosensory cortical maps within certain constraints (Allard et al. 1991). Areas lacking their previous sensory connections were "colonized" in a couple of weeks by adjacent neural maps with active connections. Experiments conducted in the 1980s found that activation patterns in the cortex that mapped sensory input from fingers underwent a distinct and orderly shift when a finger was removed. When the middle finger was removed, the neural spaces designated for the ring and index finger would enlarge, taking over the dysfunctional middle-finger map, in effect, compensating for the loss of the finger (Fox 1984). What is interesting about these studies is that this physiological "colonization," the process by which latent neural structures are appropriated and new aspects of brain

\footnotetext{
${ }^{7}$ Ibid., 50.

${ }^{8}$ Ibid., 22.

${ }^{9}$ Ibid.
} 
architecture are utilized, coincides with a type of behavioral novelty, the ability to conduct new activities in light of unprecedented environmental circumstances. Freedom-within-constraint, that odd disposition of Kantian imagination and Peircean inquiry, shows itself in dynamic neural development. This fact may indicate that the novelty of experience and inquiry are grounded in the architecture and function of the neural circuitry of our nervous systems.

In the growth and development of multimodal processes, the neural maps continue to adapt and "learn," taking advantage of the latent organizational possibilities of their constituent systems. This process of "learning" is unique to the particular physical structures of individual brains. Imaginative originality shows itself at multiple levels of analysis: Organisms perform new and imaginative functions by virtue of, and in tandem with, the novel neuronal organization that obtains in their physical embodiment. As Jerome Feldman recently writes, "mental connections are active neural connections." (Feldman 2006, p. 105) At first glance, this point may seem to be overstated in at least one respect: mental connections are accompanied by a particular quality of feeling that can not be reduced to the quantitative study of neural activation. Feldman admits that "the pleasure of beauty, the pain of disappointment, and even the feeling of being alive do not seem to us like they are reducible to neural firings and chemical reactions."10

I take Feldman's main point, however, to be that mental connections depend upon, and emerge from, neural activation. Mind is an aspect of biological and neurophysiological rhythm. A recognition of this dependence will force us to revise our epistemic and metaphysical assumptions. His comment encourages us to amend at least two long-standing epistemological commitments-our commitment to Cartesian mentalism and our common understanding of materialism. First, in terms of revising the effects of our Cartesian legacy, the discoveries associated with neuronal organization suggest that our imaginative lives are inextricably connected to our embodied lives, to the patterned relations that obtain in our physical makeup. Second, what we discover upon a close investigation of this makeup is that the spontaneity, mediation, and plasticity that we historically associated with the creation of fine art and refined thought are demonstrated at the level of material organization and processes. It is in this sense that the current study of cognitive neuroscience seeks to amend the doctrine of materialism.

\section{Imaginative adaptation and mirror neurons}

The architecture of the brain, while providing an enormous degree of variability and possibility, sets constraints on the development of certain activation patterns. The structure of the brain, in effect, sets the stage for future activation that is both free and constrained. Just as human inquiry adjusts its scope and direction over time within determinate constraints, the size and boundaries of neural maps can be changed in light of novel environmental conditions, experience-dependent learning,

\footnotetext{
${ }^{10}$ Ibid., 331.

型 Springer
} 
and social interactions. This similarity between pragmatic inquiry and neuronal adaptation is not incidental, but rather makes sense of Peirce's claim that the development and growth of thinking must arise from the development of cerebration. To make the same point in a different key, researchers are beginning to identify the physiological basis of the adaptive thinking and imaginative coordination that has so often been described phenomenologically in the accounts of classical American pragmatists.

In the late 1990s, Victorio Gallese, Giacomo Rizzolatti, and others began to investigate what would later be called the mirror neuron system. Their main goal was to expose the neural mechanisms by which primates and human beings might understand and imitate particular actions. As Umilta et al. summarize, the mirror neuron hypothesis asserts that there must be neural systems,

that recognize the actions of others. This recognition is achieved by matching the observed action on neurons which motorically coded the same action. By means of such a neural matching system, the observer during action observation is placed in the same "internal" situation as when actively activating the same action. (Umilta et al. 2001)

Exploring this hypothesis, researchers identified a set of neurons in the pre-motor cortex of humans and some primates that provide the capacity for near instantaneous response on an unconscious level both to external and internal cues. Through a mapping of particular brain areas, researchers discovered nerve nets that were activated both by the subject's observation of a meaningful action as well as by the actual performance of the action. ${ }^{11}$ For example, the same neuronal activation occurs when one grips the handle of a hammer and when one sees another person gripping a hammer in a similar way. It could be said that the social realm in which emotions are embodied and action takes place, very literally, gets under one's skin. In a colloquial sense, neuronal activation does not make a distinction between the actions and intentions of another and the actions and intentions of oneself.

The crucial point here is that these neural nets are unique in their ability to respond to, to be activated by, and to anticipate, what comes next through the subject's observations of complicated procedures. Again, to speak loosely, the neurons anticipate, and react to, the agency of others just as they would anticipate the agency of oneself. These findings are important for many reasons, but perhaps most notably in the way that they revise the standard understanding of neurophysiology and neural activity. Neural activity should not be regarded as a mechanical process delimited by a particular skull, enclosed in a particular black box. Such activity is always already "out there" - that is to say, always already in the world - responding, coordinating, mirroring the dynamics of a natural environment. At first glance, it might seem that the evidence indicates that this mirroring occurs only between animate agents or social individuals. This is only partially true. It should be remembered that these agents are a part of nature and, indeed, constituted by its dynamic processes. It is in this sense that the mirror neuron system allows us to mirror our natural environment.

11 Ibid. 
Three additional points need to be made in regard to the mirror neuron system that are, at the very least, suggestive in our discussion of the imagination and human cognition:

1) Gallese's work on the mirror neuron system indicates that similar neural activity is found in human beings when they perform an action and when they imagine or think about doing that selfsame action. This fact seems to point to the neurophysiological basis of learning by way of experiential priming. Interestingly, the visual stimuli most effective in triggering these mirror neurons were the subject's observations of actions "in which the experimenter's hand or mouth interacted with objects." (Gallese 1996) From an evolutionary perspective, this should come as no real surprise; the mouth and hands are obviously crucial in the acquisition of food and integral to the sociality of most mammals.

2) Recent studies conducted by Kohler et al. demonstrate that neurons in the ventral premotor cortex (F5) of macaque monkeys fire and are suppressed both when the animal performs a specific action and when it hears a related sound. Most of the neural nets also discharge when one observes or hears the actions of another organism performing this activity (Kohler 2002). This work indicates that partial stimuli have the ability to cause more general neural outputs. The sound of a peanut cracking generates the neural outputs that occur when an animal performs the action of cracking a peanut and when the macque observes another animal cracking the nut. These studies highlight the way in which the understanding of the actions, traditionally framed as intra-personal and insular, might arise in and through that body's creative interaction with the socialenvironmental sphere.

3) By extending these studies, Umilta et al. hypothesized that neural output associated with the performing of certain actions could be produced in animals by allowing them to observe only a small snippet of that action being performed. Their tests that employed two basic experimental conditions supported this hypothesis. In the "full vision" condition, a macque was allowed to observe an action directed toward an object. In the "hidden condition," the macque was allowed to observe the same action, except that in this case the final critical stage of the action (hand-object interaction) was shielded from the subject's view. In both conditions, the output in the mirror neuron system was largely the same, indicating that such neural responses might allow me to, in Umilta's words, "know what you are doing" even under conditions defined by partial information. ${ }^{12}$ This point sheds light on the way that bodily comportment and neuronal architecture might grant the possibility of hypothesis formation and resonates closely with Peirce's understanding of theorematic reasoning which proceeds to a conclusion that is not prefigured in particular premises.

The research on the mirror neuron system is significant in our investigation of the imagination in the sense that it begins to point to a physiological process that allows organisms to be in touch with their local situations, make generalizations from partial observations, and to adapt to their particular circumstances in the continuous

\footnotetext{
12 Ibid.

照 Springer
} 
flow of inquiry, learning and adaptation. Our discussion of the imagination has underlined the way in which it allows us to "grasp" and "handle" the novel circumstances that our surroundings afford. Additionally, the imagination, hitherto described in the sections on abduction, has a central role in our ability to make new generalization from partial cases. In the addressing the studies on the mirror neuron systems, we begin to investigate the physiological basis of these imaginative abilities.

\section{Neural reentry and imaginative coordination}

We have begun to describe the embodied character of the imagination. In turn, the emergent, and adaptive disposition of the imagination has been shown to arise from the physical/neurological processes of a physical organism. It seems necessary to more fully describe the neural processes by which various environmental stimuli (input) establish neural patterns, but also give rise to creative variations. In effect, we turn our attention back to the neurons that constitute significant parts of the brain and ask how their aggregation and selection structure the relations of an adaptive mind. In Tucker's work, it is sometimes easy to forget that the "shell" of the brain is not simply a self-contained entity, but rather constituted by relations of an estimated ten billion interconnected neurons. Each neuron receives synaptic input from many thousands of other neurons such that within each cubic millimeter of brain gray matter there are an approximate one billion synapses. We must think through this structural complexity in thinking through the imagination.

I will focus on the concept of neural reentry and degeneracy in order to examine the aggregation and selection of complex neuronal populations. ${ }^{13}$ These concepts lead to an investigation of complexity in a broader biological setting. Here we ought to regard a complex system as "one in which smaller parts are functionally segregated or differentiated across a diversity of functions but also as one that shows increasing degrees of integration when more and more of its parts interact." (Edelman and Gally 2001) Reentry and degeneracy begin to explain the way in which neural dynamics are characterized by this differentiation and integration. More distantly, they point to the process known as autopoeisis and complex agency that will be addressed in the coming sections. Any description of reentry and degeneracy should be regarded as another articulation of cognition that seeks-once again - to overcome the legacy of the Cartesian divide between matter and mind. In this respect, this rendering is another attempt to deepen an understanding of imaginative processes described earlier. In all of these discussions, we should listen for the echoing of Peirce and even Kant; their comments on the nature of the imagination seem to resonate closely with these contemporary empirical accounts.

In the previous sections, we addressed the correlation of selective events across various maps of the brain. This process has been described as being driven by the mechanism of neural reentry, a concept that rests at the heart of Edelman's research. Recognizing that the primary consciousness of human beings is characterized by an

${ }^{13}$ The concept of reentry has been addressed by many researchers for nearly two decades. See Sporns et al. (1991). Also Edelman (1997). 
integrated Gestalt that continually adjusts to one's surroundings, Edelman describes a process of neural integration that might begin to ground this conceptual continuity.

As the work of Sporns, Edelman and Tononi indicates, reentry may, at first glance, resemble a kind of biological feedback between brain regions, but differs from feedback in several important respects. Feedback (here it might be helpful to think of the workings of a mechanical thermostat) operates along, and as, a single fixed loop made of reciprocal connections using previous instructionally derived information of control and correction (known as an error parameter). In contrast, reentry is a selectional and distributed rather than an instructional system which coadapts over time without a specific and predetermined error parameter. Reentry occurs across multiple parallel pathways, connecting multiple synaptic maps, and provides for the co-evolution of these maps and connections over time (Edelman and Tononi 2000b, p. 48).

The most important distinction to be made between feedback and reentry is the fact that reentry has a constructive and reconstructive function rather than the merely corrective one that is demonstrated in feedback. Reentry is constructive in the sense that it coordinates functionally separate neural maps in developing new neural activation patterns and in reestablishing and refining preexisting patterns. Reentry appears to reflect the evolutionary and imaginative dynamics that proceed from past forms while extending them in novel ways. That is also to say that reentry is directed and irreversible. Here we are pressing on an issue that Peirce addresses in his work on continuity and generality. As mentioned earlier, in the "Law of Mind" Peirce states that "logical analysis applied to mental phenomena shows that there is but one law of mind, namely that ideas tend to spread continuously and to affect certain other ideas which stand to them in a particular relation of affectability." ${ }^{14}$ With reentry, we begin to explore the physiological basis of an idea's tendency to "spread continuously" over time. The concept of reentry trades on a question that Peirce asks in the 1890s: "What can it mean to say that ideas wholly past are thought of at all any longer?..How can a past idea be present?" Researchers are beginning to answer Peirce by identifying physical recurrent processes that underpin the continuity of human thought. The neural process that Edelman outlines in reentry is a recursive one that mediates between past patterns of activation and adaptive structural developments that respond to the novel and problematic aspects of environmental conditions as they arise. Edelman suggests that reentry is the neurophysiological foundation of the "remembered present" that defines human consciousness. The recursive activation of neuronal systems allow organisms that act in the moment, and in a particular problematic situation, to redeploy patterns of behavior that remain continuous with past forms. In terms of reentry's function in the development of human conception, it reconstructs, literally "piles together again," the various qualities and aspects of our perceptual fields in the coherence of primary consciousness. This coherence, however, does not preclude the possibility of novel forms of categorization and coordinated motor responses that arise in light of, and seek to respond to, surprising environmental conditions.

\footnotetext{
${ }^{14}$ CP 6.104 .

Springer
} 
The process of neural reentry might be understood more easily through an analogy to the development of improvisation in a group of musical artists. Imagine a jazz quartet in which each player responds to ongoing cues of her own playing, but also the cues and tempo of her accompanying players. No sheet music is used, and in the opening moments of the first movement the styles and tempos are organized around a general theme. Over time, a more specific beat and theme are established as the musicians begin to correlate, or "get in time." If the musicians have been playing together for many years, certain musical signals seem to instantaneously connect the four musicians, causing a deepening of the correlation and resonance of sound. Each new signal causes a wave of novel sounds that, in the midst of novelty, maintains harmony with past forms. As Edelman and Tononi conclude from a similar analogy: "Such integration would lead to a kind of mutually coherent music that each one acting alone could not produce." ${ }^{15}$ They elaborate on this metaphor, stating that just as two pieces of music are never identical, the dynamics, relations and connections of two brains are never exactly alike. Reentry begins to point to a mechanism that might enable this integration. In addition to being crucial to signal integration, "specific linkages of a reentrant type between two sets of groups can lead to the emergence of new associative functions not originally in either set of groups." (Edelman 1987a)

This analogy between reentry and music is instructive in two significant respects. First, it provides a way of understanding the synchronization of reentry-the reciprocal and parallel signaling that underpin the process. More significantly, this particular analogy of musical improvisation sheds light on the nature of the imagination. Musical improvisation, the beautiful give and take of embodied artists, has just been described in brief. Novelty and continuity are its defining marks. Underlying this aesthetic improvisation is embodiment - the physical processes of the musicians that grant the possibility of imaginative cognition. These processes themselves, including the process of neural reentry, help to explain the reciprocal Spiel that has long defined the realm of aesthetic judgment and production. Indeed, these neural-biological processes are best described by way of analogy to the phenomenological accounts of artistic creation. The imagination is seen once again, not as being a peculiar aspect of human aesthetics, but rather as an aspect of the very organization through which conscious life arises. ${ }^{16}$

Edelman elaborates on the concept of neural reentry, noting that it depends upon the anatomical precondition of "the remarkable massively parallel reciprocal connection" of the brain areas (Edelman 1999). This parallel, reciprocal-and one might add "mediating" - character of brain processes is exemplified by the corpus callosum, a huge bundle of reciprocal fibers that connect the two cortical hemispheres. This structural bridging, dramatically demonstrated in the corpus callosum, takes on a variety of complex forms that constitute the structure and function of the brain; other parts of the brain are coordinated by similar means. In

\footnotetext{
15 Ibid., 49.

16 Ibid.
} 
the case of the corpus callosum, the right and left hemisphere synchronize in an ongoing, recursive interchange. Reentry depends on, and is defined by, this reciprocal interchange of parallel signals between connected areas of the brain that coordinates the activities of these area's maps. As Edelman notes:

The most obvious abnormality in people with split brains...is a profound deficit in the interhemispheric integration of visual and motor information. These persons are not able to integrate visual information presented to their two visual half fields... (Edelman and Tononi 2000b, pp. 104-105)

In this sort of disorder, the reentrant mappings that usually serve to reciprocally connect functionally distinct brain regions are severed. This physiological severing causes a person's conceptual space to be torn asunder in particular ways. Our discussion of degeneracy in the coming section will readdress this situation and explain why such injuries do not cause global cognitive dysfunction.

As Edelman suggests, reentry and its anatomical substrate are crucial in the development of conceptual integration that is essential for the creation of a cohesive scene in primary consciousness. "Integration can best be illustrated," Edelman writes, "by considering exactly how functionally segregated maps in the cerebral cortex may operate coherently together even though there is no superordinate map or logically determined program." "If the experience of making music, gardening, or other creative events is multifaceted, holistic and continuous, there must be a corresponding neural integration that serves the condition for the possibility of this experience.

\section{Degeneracy and neural plasticity}

In describing the mechanism of reentry it is also necessary to address the neural property of degeneracy, mentioned in passing earlier, which seems to demonstrate the imaginative and probabilistic nature of synaptic operations and points to the physiological basis of functional adaptation. Perhaps more distantly, the concept of degeneracy may help to explain the notion of conceptual flexibility and metaphor. Research on degeneracy has shed more light on the plasticity and complexity that defines neuronal systems. In common parlance, the term degeneracy reflects a particular negative connotation as being deficient or being degraded. Researchers in general biology and cognitive neuroscience are employing the term somewhat differently, drawing on its meaning from differential calculus. In this context, degenerate equations are those that possess a common solution. As Edelman and Tononi explain in their application of degeneracy in studies of biology, "degeneracy is reflected in the capacity of structurally different components to yield similar outputs or results." 18

Degeneracy should not be confused with redundancy that occurs when structurally identical elements produce the same result. Edelman and Gally underline

\footnotetext{
${ }^{17}$ Ibid., 114.

${ }^{18}$ Ibid., 86-87, 97-98. See also Tononi et al. (1995).

글 Springer
} 
this point in their remarks concerning the difference between creation by design and creation by selection:

The contrast between degeneracy and redundancy at the structural level is sharpened by comparing design and selection in engineering and evolution, respectively. In engineering systems, logic prevails, and, for fail-safe operation, redundancy is built into design. This is not the case for biological systems...In general, an engineer assumes that interacting components should be as simple as possible, that there are no unnecessary or irrelevant interactions, that there is an explicit assignment of function...to each part of a working mechanism, and that error correction is met by feedback...By contrast, in evolutionary systems, where there is no design, the term "irrelevant" has no a priori meaning. It is possible for any change in a part to contribute to overall function. (Edelman and Gally 2001)

They elaborate on these points by claiming that, unlike the structures of engineering, the structures of evolution are not assigned exclusive responsibility for a particular function. This is also to say that evolutionary systems are defined not necessarily by simplicity, but by the propagation of the complex interactions of their parts. The flexibility and compensatory effects of degeneracy are seen in many organic systems. For example, many different DNA sequences can specify the same amino acid. Different subsets of genes can cause the same phenotypic structure. Different antibodies can identify and counteract the same foreign body.

Due to the complexity of neuronal populations of the human nervous systemcited in the section on reentry-its degree of degeneracy is far more extreme than forms examined in other cellular or genetic systems. Despite the extremely large number of neurons in most vertebrate nervous systems, no two neurons are exactly alike. Even in genetically identical organisms, no two neurons are morphologically identical. This being said, morphologically different structures can perform the same function just as many structurally dissimilar keys can open the same door.

Think about an apartment complex with many different apartments and many different tenants. Each tenant needs a key that opens her apartment and her apartment only. Each key would be structurally unique to serve this particular function. Additionally, however, every tenant would also need access to the laundry room and the room in which the trash-recycle cans are kept. Instead of producing redundant keys that opened these two rooms and distributing them to the tenants, the apartment manager makes a decision to produce a degenerate key system in which the apartment keys (remember that these must be structurally different) also open the two public rooms. Hence, different structures perform the same function. Now let us consider the advantages of this degenerate system.

The first advantage has to do with the durability of the degenerate system. Let us think about the laundry room key example. If there were only one key that could open the laundry room door, meaning if there was a one-to-one correlation between the morphology of the key and the function of opening a particular lock, this would be a fragile system indeed. If this one key was lost, or destroyed, or altered in the course of mutation, the function would be cut out of the repertoire of activities the system could undertake. Nobody would get their laundry finished. Now, imagine if getting one's laundry finished was a vital function for the system (perhaps for some of us it truly is). If this were the case, a single mutation or misplacement of the key 
would have disastrous, smelly, or even lethal, consequences. Let us say that we want to avoid this situation. We could go about it in two distinct ways. First, we could create redundant keys that opened the same lock. Very good. Now all of us can open the door and if one key is lost, damaged, or altered by mutation we need only find another copy of the key. There is, however, a cost to redundancy: resources have to be allocated for the construction of these multiple copies. There is a solution that might be more viable and would be favored in terms of evolutionary advantage - the degenerate solution. In this case, the keys would be made so as to be morphologically dissimilar yet have the same basic function of opening the public room. One might wonder how this solution would have any advantage over a redundant one.

The answer, I believe, brings us to the second advantage of degeneracy, the advantage of the flexibility and novel adaptation which degeneracy affords. In a degenerate system the morphological dissimilarity or diversity has an interesting value, a value in posse and in actu. In light of new environmental conditions these diverse forms can be put to more specific uses, oftentimes with dramatically positive consequences. For example, my key will allow me to open the public room, but due to its higher degree of structural differentiation (the extra little notch that is made on the end of the key) I am also able to perform another function, namely, opening my own apartment. This is an advantage that redundancy could never claim. The surplus of differentiation and diversity has a potential advantage to a system. It is worth noting that adaptive systems, such as the ones that operate under the rules of natural selection or Neural Darwinism, could both generate this diversity and benefit from the degenerate properties that such complexity yields. On the other hand, degenerate flexibility is notably missing in the instructional repertoire of most artificialcomputational systems.

While the laundry room key example might be helpful for certain purposes, it ought to be accompanied by two caveats. First, I would like to take a closer look at the structural character of keys. We usually regard a key as a single thing that hangs on a key chain, used for a certain purpose. The ability of a single key to function properly, however, depends on the particular configuration of its constitutive parts that make up its ridges and valleys. An infinite, but determinable, variety of configurations could possibly produce the same output or resultant function. That is not to say, however, that any configuration will produce the same function.

The second cautionary word stems from the first. If I ask you: "What opens that lock?" You could simply and easily say: "That key." It is normal to think of one key opening one lock. Degeneracy, however, problematizes this question by suggesting that many different keys or pathways could open the self-same lock-not to mention all of the hairpins, skeleton keys, credit cards and pick axes that could "unlock" the laundry room door.

On the synaptic level, degeneracy is seen when a particular environmental stimulus (or firing from another part of the brain) causes any set of neural circuits to fire in a set of output responses similar to those that were previously adaptive and provides the basis for repeated mental or physical acts. More simply, similar circuitry response can recreate specific mental or physical acts over time. Similar output responses refer to a relational similarity in which the relations between particular neural groups tend to be roughly, or approximately, the same. Edelman 
elaborates: "So what is called forth when an act is repeated must be any one or more of the neural response patterns adequate to that performance, not some singular sequence or specific detail." 19 This is a critical point. He explains that when it comes to selective cognitive systems, "there is no unique structure or combination of structures corresponding to a given category or pattern of output. Instead more than one combination of neuronal groups can participate in more than one signaling function." (Edelman and Tononi 2000b, pp. 86-87) Degeneracy may help explain how, in an ever-changing environment, the embodied mind has the ability to develop a stable, albeit flexible, pattern of behaviors and categorizations.

At first glance, the recategorization and generalization that coincide with reentry and degeneracy, however, seems to have particular limitations. How do genuinely new concepts emerge - as they do in a child's cognitive development or in the genius's artistic development? Edelman responds to this problem in somewhat vague terms. He states that "when changes in synaptic efficacy occur in neural systems... they allow the possibility of further refinement or alteration of perceptual categorization."(Edelman and Gally 2001) He attempts to address this question in more concrete terms when he turns his attention to the complexity of the reentrant interactions of neuronal groups. Neuronal groups are connected to a vast number of other groups regardless of spatial proximity. These many groups are reciprocally connected and functionally distinct from the rest of the brain. Due to this connectivity, small changes in environmental situations can cause new conceptual associations to be made and new behaviors to arise since "any subtle change in activity of different regions of the brain can bring about new, dynamic associations."(Edelman and Tononi 2000b, p. 149) Depending on the consequences of these associations, depending the way in which these associations might enable an organism to negotiate its ever-changing environment, these novel constructions may be reinforced and "learned" as they impress themselves on the dynamics of neural organization. Creative novelty does not depend on a certain supernatural élan vital that animates matter, but rather on the massively complex interactions between natural agents that grant the possibility of nonlinear effects. In discussing reentry and degeneracy, one is at least indirectly discussing the character of human memory. While the current project cannot afford a detailed treatment of this point, it does seem to be a worthwhile prompt for future investigation. Degenerate and reentrant neural circuits allow for changes in memories and concepts as new experiences occur and a new environmental context evolves. Memory, in a degenerate-reentrant system is "recategorical," re-creative, or imaginative.

\section{Conclusion: The imagination, complexity, and cognitive science}

'Imagination' shares with 'beauty' the doubtful honor of being the chief theme in esthetic writings of enthusiastic ignorance. More perhaps than any other phrase of the human contribution, it has been treated as a special and self-

19 Ibid., 98. 
contained faculty, differing from others in possession of mysterious potencies.

(Dewey 1986/1934)

\section{John Dewey Art as Experience. (1934)}

The imagination is not a "special or self-contained faculty." Indeed it is not a faculty at all. Following the lead of Dewey and other naturalists, I have argued that the imagination is rather an aspect of very organization and emergence of conscious, organic life. This argument has been advanced by the study of the neural dynamics that underpin the immediate experiential dimensions of the imagination which include spontaneity, adaptability, plasticity, and indeterminacy. These dimensions of experience have long been the primary subjects of phenomenology and have only recently become the focus of empirical science. My approach has attempted to broaden the rendering of the imagination beyond its strictly aesthetic description, to place the concept of the imagination at the heart of embodiment studies, and to maintain that a careful investigation of cognitive science may help "flesh out" a concept that has, by definition, been difficult to define.

By suggesting that the dynamics of mind tout court reflect a character that has been historically reserved for the rendering of the aesthetic imagination, I have implicitly issued a challenge to contemporary cognitive neuroscience: Cognitive science must refine its methodology and expand its focus in order to shed light on the metaphysical insights of recent embodied phenomenology. It must be refined in order to enrich, rather than reduce, the forms of human creativity. This challenge is not new. Varela's Embodied Mind (1992) outlined this challenge in the development of his neurophenomenology. His detailed studies of biological complexity in the 1970s and 1980s allowed him to articulate this challenge in clear and convincing prose (Varela 1985). Complexity, its study and its formulation, is still the most promising link between cognitive science and the phenomenological tradition. The study of the embodiment of the imagination by way of neural reentry and neural degeneracy points to this fact. Edelman's account of neural reentry and degeneracy are descriptions of complex biological dynamics. He is not interested in the biological correlates of consciousness, at least not in the sense that a correlate is a thing brought into relation with another thing. Edelman is interested in the way that "matter becomes imagination," that is to say, in the nonlinear processes of embodied thought.

If complexity is the connecting link between the empirical sciences and phenomenology, it is currently a loose link that today's scholars can tighten and strengthen. The resources are readily available for this undertaking. Neurophenomenologists ought to familiarize themselves with recent studies of complex adaptive systems (cas) such as those conducted by Stuart Kaufman and John Holland (Kauffman 2000; Holland 1995). These researchers provide lucid accounts of complexity in the natural world, accounts that may provide the theoretical frame for future investigations of the imagination by cognitive neuroscience. If the imagination is "in possession of mysterious potencies," it is only through the studies of complexity that science can handle, and work through, this riddle of the embodied mind. 


\section{References}

Allard, T., et al. (1991). Reorganization of somatosensory area 3b representations in adult owl monkeys after digital syndetyly. Journal of Neurophysiology, 66, 1048-1058.

Dennett, D. (1988). Quining Qualia. In A. J. Marcel, \& E. Bisiach (Eds.), Consciousness in contemporary science. Oxford: Oxford University Press.

Dewey, J. (1986/1934). Art as experience. In J. A. Boydson (Ed.), John Dewey: The later works, 19251953 (vol. 12 (p. 271). Carbondale: Southern Illinois University Press.

Edelman, G. (1987a). Neural Darwinism: The theory of neuronal group selection. New York: Basic Books.

Edelman, G. (1987b). Neural Darwinism. New York: Basic Books.

Edelman, G. (1989). The remembered present: A biological theory of consciousness p. 50. New York: Basic Books.

Edelman, G. (1997). Neural dynamics in a model of the thalamocortical system, 2: The role of neural synchrony tested through the perturbations of spike Timing. Cerebral Cortex, 7, 228-236.

Edelman, G. (1999). Building a picture of the brain. Annals of the New York Academy of Science, 882(1), 70.

Edelman, G., \& Gally, J. (2001). Degeneracy and complexity in biological systems. Proceeding of the National Academy of Sciences, 13767, (Nov. 6).

Edelman, G., \& Tononi, G. (2000a). A universe of consciousness: How matter becomes imagination. Cambridge: Perseus Books.

Edelman, G., \& Tononi, G. (2000b). A universe of consciousness: How matter becomes imagination. New York: Basic Books.

Engberg, P. (1995). The concept of domain in cognitive theory of metaphor. Nordic Journal of Linguistics, $18,111-119$.

Engberg, P. (1999). Space and time: A metaphoric relation.Cognitive semantics: Meaning and cognition. Amsterdam: Benjamin.

Feldman, J. (2006). From molecule to metaphor. Cambridge: MIT.

Fox, J. (1984). The brain's dynamic way of keeping in touch. Science, 225(4664), 820.

Gallese, V. (1996). Action recognition in the premotor cortex. Brain, 119, 593-609.

Gallese, V., \& Metzinger, T. (2003). The emergence of a shared action ontology: Building blocks for a theory. Consciousness and Cognition, 12, 549-571.

Gallistel, C. (1990). The organization of learning. Cambridge: MIT.

Glenberg, A. (2002). Grounding language in action. Psychonomic Bulletin and Review, 9, 558-565.

Hauk, O. (2004). Somatotopic representations of action words in human motor and premotor cortex. Neuron, 41, 301-7.

Holland, J. (1995). Hidden order: How adaptation builds complexity. Cambridge: Perseus Books.

James, W. (1950). The principles of psychology p. 262. New York: Dover Press.

Johnson, M. Dewey's Zen: The 'oh' of wonder. Discussion paper at the Society for the Advancement of American Philosophy.

Johnson, M., \& Lakoff, G. (1999). Philosophy in the flesh: The embodied mind and its challenge to western thought. New York: Harper Collins.

Kauffman, S. (2000). Investigations. Oxford: Oxford University Press.

Kohler, E. (2002). Hearing sounds, understanding actions: Action representation in mirror neurons. Science, 297, 846.

Kohler, E., Gallese, V., et al. (2002). Hearing sounds, understanding actions: Action representations in mirror neurons. Science, 297, 846-848.

Maturana, H. (1980). Autopoeisis: The organization of the living. In Autopoeisis and cognition. Dordrecht: Reidel.

Rohrer, T. (2006). Image schemata and the brain. In B. Hampe, \& J. Grady (Eds.), From perception to meaning: Image schemas in cognitive linguistics. Berlin: Mouton de Gruyter.

Rose, S. (1987). Cross modal abilities in human infants. In J. Osofsky (Ed.), Handbook of infant development (pp. 318-362). New York: Wiley.

Sporns, O., et al. (1991). Modeling perceptual grouping and figure-ground segregation by means of active reentrant connections. Proceedings of the National Academy Science of the United States of America, $88,129-133$.

Schwartz, J., \& Begley, S. (2002). The mind and the brain: Neuroplasticity and the power of mental force p. 118. New York: Regent. 
Tononi, G., et al. (1995). Measures of degeneracy and redundancy in biological networks. Proceedings of the National Academy of Sciences of the United States of America, 96, 3188-3208.

Umilta, M., et al. (2001). I know what you are doing: A neurophysiological study. Neuron, 31, 155-165.

Varela, F. (1992). The embodied mind: Cognitive science and human experience. Cambridge: MIT.

Varela, F. (1985). Complexity of the brain and autonomy of the living. In G. Bocchi, \& M. Ceruti (Eds.), La Sfida della Complessit (pp. 141-157). Feltrinelli: Milano. 